\title{
Elasto-capillary coalescence of multiple parallel sheets
}

\author{
A. D. Gat $\dagger$ and M. Gharib \\ Graduate Aerospace Laboratories, California Institute of Technology, Pasadena, CA 91125, USA \\ (Received 11 June 2012; revised 6 February 2013; accepted 7 February 2013; \\ first published online 16 April 2013)
}

We analyse two-dimensional clamped parallel elastic sheets which are partially immersed in liquid as a model for elasto-capillary coalescence. In the existing literature this problem is studied via minimal energy analysis of capillary and elastic energies of the post-coalescence state, yielding the maximal stable post-coalescence bundle size. Utilizing modal stability analysis and asymptotic analysis, we studied the stability of the configuration before the coalescence occurred. Our analysis revealed previously unreported relations between viscous forces, body forces, and the instability yielding the coalescence, thus undermining a common assumption that coalescence will occur as long as it will not create a bundle larger than the maximal stable post-coalesced size. A mathematical description of the process creating the hierarchical coalescence structure was obtained and yielded that the mean number of sheets per coalesced region is limited to the subset $2^{N}$ where $N$ is the set of natural numbers. Our theoretical results were illustrated by experiments and good agreement with the theoretical predictions was observed.

Key words: instability, interfacial flows (free surface), low-Reynolds-number flows

\section{Introduction}

The study of the interaction between elastic and capillary forces is relevant to phenomena such as self-folding of solid sheets (commonly referred to as capillary origami, Py et al. 2007b; Pineirua, Bico \& Roman 2010; Antkowiak et al. 2011), densification of patterned arrays of carbon nanotubes (Journet et al. 2005; Huang et al. 2007; Zhao et al. 2010; De Volder et al. 2010, 2011), and self-assembly and modification of the mechanical and geometrical properties of arrays of solid structures (Chandra et al. 2009; Pokroy et al. 2009; Chiodi, Roman \& Bico 2010; Duan \& Berggren 2010; Elwenspoek et al. 2010; Kang et al. 2011).

Studies of the elasto-capillary coalescence of two elastic sheets due to capillary force of a liquid between them were made by Kim \& Mahadevan (2006), Duprat, Aristoff \& Stone (2011), Aristoff, Duprat \& Stone (2011), Duprat et al. (2012) and Taroni \& Vella (2012). Experimental studies of elasto-capillary coalescence of multiple elastic structures include the works of Py et al. (2007a), Chandra et al. (2009), Pokroy et al. (2009), Chandra \& Yang (2010), Chiodi et al. (2010), Duan \& Berggren (2010), Elwenspoek et al. (2010), and Kang et al. (2011). Previous theoretical studies of elasto-capillary coalescence (e.g. Journet et al. 2005; Zhao \& Fan 2006; Boudaoud, 
Bico \& Roman 2007; Py et al. 2007a; Chandra et al. 2009; Chiodi et al. 2010) followed the approach of Bico et al. (2004) and analysed the post-coalescence state utilizing energy minimization analysis. This approach involves studying the stability of bundles of $n$ solid structures connected by capillary forces and examining if reaching the minimal energy state requires separation of the bundle into two smaller bundles. The largest number of solid structures which do not separate, $n_{\max }$, can thus be obtained. An assumption then is made that coalescence will continue as long as it will not create a bundle of $n>n_{\max }$ solid structures. However, for large arrays of solid structures, initially, capillary forces act in all directions and there is no net force acting to deflect the solid structures. Therefore, the initial state is not included in the states described by energy minimization analysis.

Unlike previous works, we analyse the pre-coalescence configuration by studying the stability of the solid-liquid interaction before the coalescence occurred. We obtain previously unreported effects of body forces (gravity), extraction process and viscosity on the elasto-capillary instability, suggesting novel methods to control elasto-capillary coalescence. These results could not have been calculated by the existing approach which only involves a balance between elastic and capillary forces. For the case of large arrays of solid structures, our results undermine the validity of the assumption that coalescence will continue as long as it will not create a bundle of $n>n_{\max }$ solid structures.

In addition, the energy minimization analysis yielding $n_{\max }$ does not describe the process or the hierarchical structure of the coalescence, only the upper limit on the number of solid structures in a coalesced bundle. Boudaoud et al. (2007) studied the aggregation process of flexible strips progressively withdrawn from a liquid bath. As the strips are withdrawn from the liquid their effective length increases with time, thus changing the elastic forces and turns $n_{\max }$ into a function of time $n_{\max }(t)$. Coalescence is then assumed to occur until $n>n_{\max }(t)$, yielding an hierarchical coalescence structure due to the time dependence of $n_{\max }$. Pokroy et al. (2009) studied self-organization of arrays of bristles into hierarchical assemblies by capillary forces and experimentally observed multistep sequential coalescence of the small bundles into larger bundles of solid structures (i.e. initially bundles of two structures would be created, then bundles of four structures, followed by bundles of eight structures, etc.). While hierarchical structures can be created by time-varying capillary or elastic forces (as Boudaoud et al. 2007 suggest) our results yield that the hierarchical structure will be created even without such time-varying $n_{\max }(t)$. Our model (via modal stability analysis) describes the process which creates the hierarchical coalescence structure by calculating the growth rates of perturbations with different wavelengths, yielding that the mean number of sheets per coalesced region is limited to the subset $2^{N}$ where $N$ is the set of natural numbers and a multistep coalescence process (in agreement with the experimental observations of Pokroy et al. 2009).

Similarly to previous studies (e.g. Kim \& Mahadevan 2006; Liu et al. 2007; Chiodi et al. 2010; Duprat et al. 2011) we focus on clamped parallel elastic sheets which are partially immersed in liquid as a model of elasto-capillary coalescence of multiple solid structures. The rest of this paper is organized as follows: In $\S 2$ we utilize the lubrication analysis and linear beam theory to model the interaction between the liquid and the solid sheets. In $\S 3.1$ we apply modal stability analysis to study the stability of the configuration before the coalescence occurred and estimate the mean number of sheets per coalesced region. In $\S 3.2$ we experimentally illustrate our theoretical results and in $\S 4$ we present concluding remarks. 
(a)

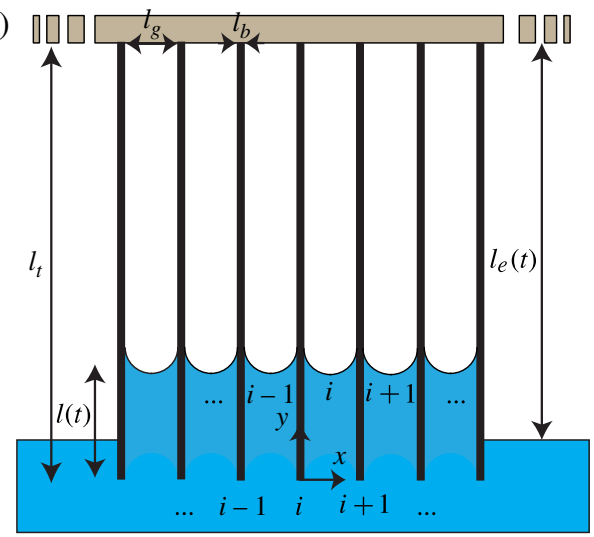

(b)

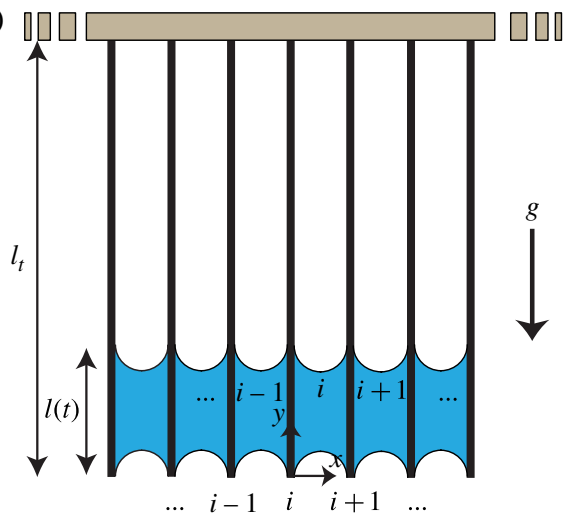

FIgURE 1. (Colour online) Schematic description of multiple parallel elastic sheets immersed within a liquid before $(a)$ and after $(b)$ extraction from the liquid. The initial gap between the sheets is $l_{g}$, the thickness of a single sheet is $l_{b}$, the length of the sheets in the $y$-direction is $l_{t}$, the length of the wetted region is $l(t)$. The origin of the Cartesian coordinate system is defined at the lower end of beam $i$.

\section{Analysis}

\subsection{Problem definition}

We focus on the dynamics of multiple two-dimensional parallel elastic sheets which are initially immersed (see figure $1 a$ ) and then extracted from a liquid (see figure $1 b$ ). Due to capillary forces the liquid rises into the lower part of the sheets. As the sheets are extracted from the liquid, liquid bridges remain and connect the elastic sheets (see figure $1 b$ ). The pressure field within the liquid and the lateral meniscus forces at the three-phase contact line act on the elastic sheets and may deform them, which in turn can affect the pressure applied by the liquid due to capillary, gravity, and viscous forces. This interaction can yield coalescence of the elastic sheets into bundles containing multiple elastic sheets. We limit our analysis to cases in which inertia of both the solid and liquid is negligible and $N \gg N_{s}$, where $N$ is the total number of sheets in the array and $N_{s}$ is the number of sheets within a single collapsed bundle.

The geometry of the array is defined by $l_{t}$, the length of the sheets in the $y$-direction, $l_{g}$, the gap between the sheets at the clamped end, $y=l_{t}$ (see figure 1), $l_{b}$, the thickness of a single sheet, $l_{z}$, the length of the sheets in the $z$-direction, $l(t)$, the length of the wetted region of the sheet, $l_{e}(t)$, the distance between the clamped end of the sheet and the surface of the liquid and $w_{i}(y, t)$, the deflection of sheet $i$. The physical properties of the sheets are $E$, the Young modulus, $I$, the second moment of inertia, and $m$, the mass per unit length. The physical properties of the liquid are, $\rho$, its density, $\mu$, viscosity, $g$, gravity, $\gamma$, surface tension, $\theta$, the wetting angle, $p$, the pressure, and $(u, v)$, liquid velocity.

We define the small parameters

$$
\varepsilon_{1}=\frac{l_{0}}{l_{t}} \ll 1
$$

and

$$
\varepsilon_{2}=\frac{l_{g}}{l_{0}} \ll 1,
$$


where $l_{0}$ is the characteristic height of the liquid-filled region. For a fast extraction speed (compared with the characteristic speed of the flow due to capillary forces, $\left.\partial l_{e} / \partial t \gg \gamma l_{g} / 12 \mu l(0)\right) l_{0}$ can be estimated as $l_{0} \approx l(0)$, the height of the liquid before extraction of the array from the liquid bath. For slow extraction speed, $\partial l_{e} / \partial t \ll$ $\gamma l_{g} / 12 \mu l(0), l_{0}$ can be estimated by quasi-static capillary rise as $l_{0} \approx 2 \gamma \cos (\theta) / l_{g} \rho g$.

\subsection{The elastic problem}

The bending of plate $i$ can be described in terms of the Young modulus, $E$, second moment of inertia, $I$, mass per unit length, $m$, and external load applied on the sheet by the liquid, $q_{i}(y)$, by the Euler-Bernoulli equation

$$
E I \frac{\partial^{4} w_{i}}{\partial y^{4}}+m \frac{\partial^{2} w_{i}}{\partial t^{2}}=q_{i}(y)
$$

and the boundary conditions

$$
w_{i}\left(y=l_{t}\right)=0, \quad \frac{\partial w_{i}}{\partial y}\left(y=l_{t}\right)=0, \quad \frac{\partial^{2} w_{i}}{\partial y^{2}}(y=0)=0, \quad \frac{\partial^{3} w_{i}}{\partial y^{3}}(y=0)=0,
$$

corresponding to no deflection at the clamped end, no slope at the clamped end, no bending momentum at the free end and no shear force at the free end, respectively.

Hereafter all normalized variables are denoted by capital letters. We define the normalized coordinates $(X, Y)=\left(x / l_{t}, y / l_{t}\right)$, deflection of sheet $i W_{i}=w_{i} / l_{g}$, length of the region filled with liquid $L=l / l_{0}$ (see figure 1), time $T=u_{0} t / l_{g}$, and beam load $Q_{i}=q_{i} / p_{0} l_{z}$, where $u_{0}$ is characteristic sheet deflection speed. Substituting the normalized variables into the Euler-Bernoulli equation (2.3) we obtain

$$
\frac{\partial^{4} W_{i}}{\partial Y^{4}}+\frac{m l_{t}^{4} u_{0}^{2}}{E I l_{g}^{2}} \frac{\partial^{2} W_{i}}{\partial T^{2}}=\frac{l_{t}^{4} p_{o} l_{z}}{E I l_{g}} Q_{i}
$$

From (2.1), the loading of the sheet can be approximated as a point force acting at $y=0$ with accuracy of $O\left(\varepsilon_{1}\right)$. In addition, we limit our analysis to cases with negligible momentum of the elastic sheets, $m l_{t}^{4} u_{0}^{2} / E I l_{g}^{2} \ll 1$. Solving the ordinary differential equation (2.5) and applying the relevant boundary conditions (2.4) yields a linear relation between the load and the deflection at the tip of the sheet, hereafter denoted by $D_{i}$,

$$
D_{i}(T)=W_{i}(0, T)=\frac{l_{z} p_{0} l_{t}^{4}}{3 E I l_{g}} \int_{0}^{\varepsilon_{1} L} Q_{i} \mathrm{~d} Y+O\left(\varepsilon_{1}\right) .
$$

We thus define the reference pressure $p_{0}$ as $p_{0}=3 E I \varepsilon_{2} / l_{t}^{3} l_{z}$, as a characteristic pressure difference of the problem. Similarly, utilizing Taylor expansion around $Y=0$, the deflection $W_{i}(Y, T)$ in the region $0<Y<\varepsilon_{1}$ can be estimated at leading-order as

$$
W_{i}(Y, T)=\sum_{n=0}^{\infty} \frac{Y^{n}}{n !} \frac{\partial^{n} W_{i}(0, T)}{\partial Y^{n}}=D_{i}+O\left(\varepsilon_{1}\right) .
$$

\subsection{The fluidic problem}

We limit our analysis to Newtonian incompressible liquids which are governed by the Navier-Stokes conservation of momentum equations

$$
\rho\left(\frac{\partial u}{\partial t}+u \frac{\partial u}{\partial x}+v \frac{\partial u}{\partial y}\right)=-\frac{\partial p}{\partial x}+\mu\left(\frac{\partial^{2} u}{\partial x^{2}}+\frac{\partial^{2} u}{\partial y^{2}}\right)
$$




$$
\rho\left(\frac{\partial v}{\partial t}+u \frac{\partial v}{\partial x}+v \frac{\partial v}{\partial y}\right)=-\frac{\partial p}{\partial y}+\mu\left(\frac{\partial^{2} v}{\partial x^{2}}+\frac{\partial^{2} v}{\partial y^{2}}\right)-\rho g,
$$

and conservation of mass

$$
\frac{\partial u}{\partial x}+\frac{\partial v}{\partial y}=0
$$

The boundary conditions at the solid-liquid interface, $x=w_{i}(y, t)$, are no-slip, $v=0$, and no-penetration, $u=\partial w_{i}(y, t) / \partial t$. The boundary condition at the gas-liquid interface is the dynamic stress balance (Leal 2007)

$$
\left(\boldsymbol{\tau}^{*}-\boldsymbol{I} p^{*}\right) \cdot \hat{\boldsymbol{n}}-(\boldsymbol{\tau}-\boldsymbol{I} p) \cdot \hat{\boldsymbol{n}}+\gamma c \hat{\boldsymbol{n}}=0,
$$

where $\boldsymbol{u}$ is velocity, $p^{*}$ is the pressure of the surrounding gas, $\boldsymbol{\tau}^{*}$ is the viscous stress tensor of the surrounding gas, $\boldsymbol{\tau}$ is the viscous stress tensor of the liquid, $\hat{\boldsymbol{n}}$ is a unit vector pointing out of the liquid surface and $c$ is the local curvature.

Following standard lubrication theory, we denote the characteristic length scales of the liquid flow between the elastic sheets as $l_{g}$ and $l_{0}$, in the $x$-and $y$-directions, respectively. Substituting the characteristic lengths into the continuity equation and performing order-of-magnitude analysis of (2.10) we obtain

$$
\frac{u_{0}}{v_{0}} \sim \frac{l_{g}}{l_{0}}=\varepsilon_{2},
$$

where $u_{0}$, the characteristic sheet deflection speed, is equal to the characteristic liquid speed in the $x$-direction, $v_{0}$ is the characteristic liquid speed in the $y$-direction and $\sim$ denotes asymptotic equivalence.

Since the length scales characterizing the liquid flow field are different from the length scales characterizing the deformation of the elastic sheets, we define new, renormalized, coordinates $\left(X_{l}, Y_{l}\right)=\left(x / l_{g}, y / l_{0}\right)$ which are appropriate to the liquid region. We define the normalized velocity $(U, V)=\left(u / u_{0}, \varepsilon_{2} v / u_{0}\right)$, pressure $P=p / p_{0}$ (where $p_{0}$ is the characteristic pressure), gravity $G=\rho g l_{g}^{2} / \mu v_{0}$ and curvature of the gas-liquid interface $C=l_{g} c$. We denote $\varepsilon_{2} R e=\varepsilon_{2} \rho u_{0} z_{0} / \mu$ as the reduced Reynolds number. For $\varepsilon_{2} R e \ll 1$ inertia within the liquid is negligible and thus the volume of liquid within the array can be calculated from the balance between viscous and capillary forces. The dominant balance of the $y$-direction momentum conservation equation (2.9) is thus between pressure gradient and viscosity and order-of-magnitude analysis yields $v_{0}=p_{0} \varepsilon_{2}^{2} l_{0} / \mu=3 E I \varepsilon_{2}^{3} \varepsilon_{1} / l_{t}^{2} l_{z} \mu$.

Substituting the normalized variables into the governing equations, the momentum conservation equations, at leading-order, are reduced to

$$
\frac{\partial P}{\partial X_{l}}=O\left(\varepsilon_{2}^{2}, \varepsilon_{2} R e\right)
$$

and

$$
\frac{\partial P}{\partial Y_{l}}=\frac{\partial^{2} V}{\partial X_{l}^{2}}-G+O\left(\varepsilon_{2}^{2}, \varepsilon_{2} R e\right)
$$

We require that the viscosity of the gas, $\mu^{*}$, is small in comparison with the viscosity of the liquid, $\mu^{*} \ll \mu$. Thus, the dynamics of the flow of the gaseous phase can be neglected. Normalizing the boundary conditions, the dynamic stress balance at the 
gas-liquid interface, at leading-order, is thus reduced to

$$
P-P^{*}+C \frac{\varepsilon_{2}^{2}}{C_{a}} \sim 0,
$$

where $C_{a}=\mu u_{0} / \gamma$ is the capillary number and $P^{*}=p^{*} / p_{0}$ is the normalized pressure of the surrounding gas. For $\varepsilon_{2} \rightarrow 0$ and small Bond number $\rho g l_{g}^{2} / \gamma \ll 1$, the curvature at the liquid-gas interface is part of a circular arc and can be estimated from geometric relations as $C \sim 2 \cos \theta /\left(1-D_{i}+D_{i+1}\right)$ (Gat, Navaz \& Gharib 2011), where $\theta$ is the static (advancing or receding) wetting angle at the solid-liquid boundary. Thus, the pressure at the liquid-gas interface, denoted as $Y_{l}=L\left(X_{l}, T\right)$ where $L$ is the vertical location of the solid-liquid interface (see figure 1), is given by

$$
P\left(Y_{l}=L\right) \sim P^{*}-\frac{\varepsilon_{2}^{2}}{C_{a}} \frac{2 \cos \theta}{1-D_{i}+D_{i+1}} .
$$

For the case of $C_{a} \sim \varepsilon_{2}^{2}$ or $C_{a} \ll \varepsilon_{2}^{2}$, the wetting angles will approach their static advancing or receding values (De Gennes et al. 2004; Sikalo, Tropea \& Ganic 2005) and for the case of $C_{a} \gg \varepsilon_{2}^{2}$ the influence of surface tension is negligible and thus (2.16) is valid, at leading-order, for any capillary number $C_{a}$. The pressure at $Y_{l}=0$ is $P_{i}(0)=P^{*}-G\left(L_{t}-L_{e}(T)\right)$ before the extraction of the sheets from the liquid (see figure $1 a)$ or $P_{i}(0)=P_{i}\left(L_{i}\right)+G L_{i}(T)$ after the extraction of the sheets from the liquid (see figure $1 b$ ).

We focus on the flow in the region $l_{b} / 2+D_{i}<X_{l}<1+l_{b} / 2+D_{i+1}$ (which is readily generalized to all other regions) and denote the pressure in this region by $P_{i}$. The height of the liquid in this region is denoted by $L_{i}$ and can be estimated as $L_{i}\left(X_{l}, T\right)=L_{i}(1 / 2, T)+O\left(\varepsilon_{2}\right)$ and thus for $\varepsilon_{2} \rightarrow 0, L_{i}\left(X_{l}, T\right) \rightarrow L_{i}(T)$. Integrating the $Y$-momentum conservation equation (2.14) we obtain

$$
V \sim\left(\frac{\partial P_{i}}{\partial Y_{l}}+G\right)\left[\frac{\left(X_{l}-D_{i}\right)\left(X_{l}-1-D_{i+1}\right)}{2}\right]+\frac{\partial L_{e}}{\partial T}
$$

(for the case in which the sheets are extracted from the liquid, see figure $1 b$, $\left.\partial L_{e} / \partial T=0\right)$.

\subsection{Model for elasto-capillary coalescence}

Substituting (2.17) into (2.10) and integrating from $X_{l}=D_{i}+l_{b} / 2$ to $1+D_{i+1}+l_{b} / 2$, we obtain

$$
\frac{\partial D_{i+1}}{\partial T}-\frac{\partial D_{i}}{\partial T}-\frac{\left(1+D_{i+1}-D_{i}\right)^{3}}{12} \frac{\partial}{\partial Y_{l}}\left(\frac{\partial P_{i}}{\partial Y_{l}}+G\right) \sim 0 .
$$

Integrating (2.18) twice with regard to $Y_{l}$ yields the pressure field

$$
P_{i} \sim 6\left(\frac{\partial D_{i+1}}{\partial T}-\frac{\partial D_{i}}{\partial T}\right) \frac{Y_{l}^{2}-Y_{l} L_{i}}{\left(1+D_{i+1}-D_{i}\right)^{3}}+\frac{Y_{l}}{L_{i}} P_{i}\left(L_{i}\right)+\left(1-\frac{Y_{l}}{L_{i}}\right) P_{i}(0) .
$$

The force applied by capillary pressure on beam $i$ scales as $O\left(\gamma l(0) l_{z} / l_{g}\right) \sim O\left(\gamma l_{z} / \varepsilon_{2}\right)$ where the force applied by the liquid meniscus scales as $O\left(\gamma l_{z}\right)$. Thus, lateral meniscus forces can be neglected in the leading-order analysis. The force applied by the liquid on beam $i$, denoted by $F_{i}$ (and normalized by $p_{0} l_{z} l_{0}$ ), is therefore obtained at leading- 
order by integration of the pressure over $l_{b} / 2+D_{i}<X_{l}<1+l_{b} / 2+D_{i+1}$ :

$$
\int_{0}^{L_{i}} P_{i} \mathrm{~d} Y_{l} \sim \frac{L_{i}}{2} P\left(L_{i}\right)+\left(1-\frac{L_{i}}{2}\right) P(0)-\left(\frac{\partial D_{i+1}}{\partial T}-\frac{\partial D_{i}}{\partial T}\right)\left(\frac{L_{i}}{1+D_{i+1}-D_{i}}\right)^{3} .
$$

The load acting on beam $i, Q_{i}$, can be calculated by combining the pressures acting on both sides of the sheet. Substituting $Q_{i}$ into (2.6) yields

$$
\begin{aligned}
D_{i} \sim & \left(\frac{L_{i}}{1+D_{i+1}-D_{i}}\right)^{3}\left(\frac{\partial D_{i+1}}{\partial T}-\frac{\partial D_{i}}{\partial T}\right)-\left(\frac{L_{i-1}}{1+D_{i}-D_{i-1}}\right)^{3}\left(\frac{\partial D_{i}}{\partial T}-\frac{\partial D_{i-1}}{\partial T}\right) \\
& +\frac{L_{i-1}}{2}\left(P_{i-1}\left(L_{i-1}\right)+P_{i-1}(0)-2 P^{*}\right)-\frac{L_{i}}{2}\left(P_{i}\left(L_{i}\right)+P_{i}(0)-2 P^{*}\right),
\end{aligned}
$$

and we thus obtain an equation relating the deflection of beam $i$ to the deflection of beams $i-1$ and $i+1$. Since this relation can be generalized to any of the beams, we thus obtain a system of equations describing the deflections of an array of beams due to forces applied by the liquid. The trivial steady homogeneous solution of the system of equations (2.21) corresponds to the initial state of the sheets before elasto-capillary coalescence occurred, and is defined by $D_{n}=0, L_{n}=L(0)$ for any $n$.

\section{Results}

\subsection{Stability analysis}

As the sheets are extracted from the liquid the effective length of the sheets increases and thus the elastic resistance to deformation decreases. The least resistance to elastic deformation occurs after the total extraction of the sheets from the liquid. Therefore, elasto-capillary coalescence will be limited by the stability of the configuration after extraction from the liquid (which is the final state, see figure $1 b$ ). We thus focus our analysis on the stability of the configuration after extraction from the liquid.

Flow during the extraction process can affect $l(0)$ (via (2.17)), the length of the wet region before the coalescence, which will influence the capillary force and thus the elasto-capillary coalescence. For cases in which the extraction speed is fast compared with the characteristic speed of the flow due to capillary forces, $\partial l_{e} / \partial t \gg \gamma l_{g} / 12 \mu l(0)$, $l(0)$ can be estimated as the height of the liquid before extraction of the array from the liquid surface (see figure $1 a$ ). Similarly, for cases in which the extraction speed is slow compared with the characteristic speed of the flow due to capillary forces, $\partial l_{e} / \partial t \ll \gamma l_{g} / 12 \mu l(0), l(0)$ is determined by quasi-static capillary rise as $l(0)=2 \gamma \cos (\theta) / l_{g} \rho g$. For slow extraction speeds elasto-capillary coalescence can occur during the extraction process. Since the capillary rise is inversely related to the gap between the sheets, the value of the capillary rise after $n$ elasto-capillary coalescence will be $l(0) \times 2^{-n}$, similarly to the case of elasto-capillary collapse after extraction, see figure 2 below. Thus, the value of $l(0)$ computed by capillary rise before the coalescence can be used to calculate the elasto-capillary instability after extraction, even if coalescence occurred during the extraction from the liquid, since the upper limit on $N_{s}$ will be determined by the stability after extraction of a configuration with a constant liquid volume.

We investigate the stability of the initial trivial homogeneous solution in order to model the process of elasto-capillary coalescence. We introduce small perturbations $D_{i}=D_{i, 0}+\varepsilon D_{i, 1}$ around the initial solution, where $D_{i, 0}$ is the steady solution, $D_{i, 1}$ is the perturbation and $\varepsilon \rightarrow 0$. Substituting the perturbations into conservation of mass equation (2.10), the length of the liquid-filled region between sheet $i$ and sheet $i+1$, 

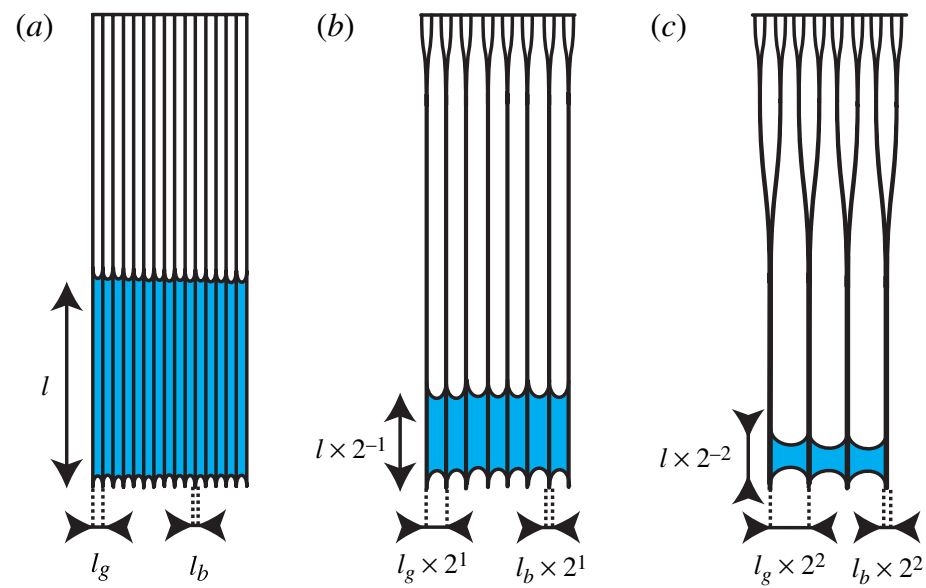

FIGURE 2. (Colour online) The effects of elasto-capillary collapse of the minimal mode on the capillary forces (via $l$ and $l_{g}$, the length of the wetted region and gap between the sheets, respectively) and elastic forces (via $l_{b}$, the width of the bundle) of the configuration. (a) Illustration of the initial state; $(b)$ the system after the fastest growing collapse mode of the initial state shown in $a$ ); and (c) the system after the fastest growing collapse mode of the configuration illustrated in $(b)$.

denoted by $L_{i}(T)$ (and normalized by $l_{0}$ ), can be described at first-order as

$$
L_{i}(T) \sim L(0)\left(1-\varepsilon\left(D_{i+1}-D_{i}\right)\right),
$$

where $L(0)$ is the length of the liquid-filled region before the coalescence. Substituting the boundary condition $P_{i}(0)=P_{i}\left(L_{i}\right)+G L_{i}(T),(3.1)$, and the above perturbations into (2.21) yields the governing leading-order equation for the perturbations,

$$
D_{i, 1} \sim\left(L^{3}(0) \frac{\partial}{\partial T}+\frac{G L^{2}(0)}{2}-L(0) \frac{\varepsilon_{2}^{2}}{C_{a}} 4 \cos \theta\right)\left(D_{i+1,1}-2 D_{i, 1}+D_{i-1,1}\right) .
$$

We thus obtain a system of $n$-equations describing the deflections of the elastic sheets. Reverting from the $\left(X_{l}, Y_{l}\right)$ coordinates system to the $(X, Y)$ coordinates, utilizing the relation $l_{g} / l_{t}=\varepsilon_{1} \varepsilon_{2} \ll 1$, and finite-differences analysis, we can approximate the term $D_{i+1,1}-2 D_{i, 1}+D_{i-1,1}$ as a second derivative of $D$ with regard to $X$, yielding the continuous equation

$$
D(X) \sim\left(L^{3}(0) \frac{\partial}{\partial T}+\frac{G L^{2}(0)}{2}-L(0) \frac{\varepsilon_{2}^{2}}{C_{a}} 4 \cos \theta\right)\left(\frac{l_{g}+l_{b}}{l_{t}}\right)^{2} \frac{\partial^{2} D}{\partial X^{2}} .
$$

Utilizing modal stability analysis, we introduce the perturbations $D \sim{ }_{\varepsilon} C \exp (\alpha T+$ $i 2 \pi X / \lambda)$, where $\alpha$ is the growth rate and $\lambda$ is the wavelength of the perturbation (scaled by $l_{t}$ ). The growth rate $\alpha$ can thus be obtained from (3.3) and is a function of the wavelength $\lambda$, defined as

$$
\alpha(\lambda) \sim \frac{1}{L^{2}(0)} \frac{\varepsilon_{2}^{2}}{C_{a}} 4 \cos \theta-\frac{G}{2 L(0)}-\frac{\lambda^{2}}{4 \pi^{2} L^{3}(0)}\left(\frac{l_{t}}{l_{g}+l_{b}}\right)^{2} .
$$

Equation (3.4) indicates that the smallest wavelength grows the fastest, and thus the minimal collapse mode (which is the collapse of two sheets, corresponding 
to $\left.\lambda=\left(l_{g}+l_{b}\right) / l_{t}\right)$ is the fastest growing instability. After the collapse of the minimal mode occurs, the geometry of the array will be modified (see figure 2). Similarly to the estimates made by Bico et al. (2004), Boudaoud et al. (2007) and Chiodi et al. (2010), we estimate the modified parameters $I_{n} \sim I \times 2^{n}, l_{g, n} \sim l_{g} \times 2^{2 n}, l_{b, n} \sim l_{b} \times 2^{n}$ and $l_{0, n} \sim l_{0} \times 2^{-n}$, where $I_{n}, l_{g, n}, l_{b, n}$ and $l_{0, n}$ are the second moment of inertia, gap, and width of the coalesced regions and length of the liquid-filled region, respectively, after $n$ coalescences. Some of the dimensionless parameters based on these values are thus modified as well. We denote $C_{a, n} \sim C a \times 2^{8 n}, \varepsilon_{2, n} \sim \varepsilon_{2} \times 2^{2 n}, G_{n} \sim G \times 2^{-4 n}$, as the capillary number, shallowness parameter of the liquid, and normalized gravity after $n$ coalescences, respectively. Substituting these into (3.4) yields the equation governing the growth rate of perturbations with wavelength $\lambda$ after $n$ collapses, denoted as $\alpha_{n}$,

$$
\alpha_{n}(\lambda) \sim 2^{-4 n} \frac{1}{L^{2}(0)} \frac{\varepsilon_{2}^{2}}{C_{a}} 4 \cos \theta-2^{-4 n} \frac{G}{2 L(0)}-2^{-2 n} \frac{\lambda^{2}}{4 \pi^{2} L^{3}(0)}\left(\frac{l_{t}}{l_{g}+l_{b}}\right)^{2} .
$$

The minimal collapse mode after $n$ occurrences of the coalescence instability is $\lambda \sim 2^{n}\left(l_{g}+l_{b}\right) / l_{t}$. Therefore, for elasto-capillary coalescence to occur after $n$ previous collapses (which will create the $n+1$ collapsed state) it is required that

$$
\alpha_{n}\left(\lambda \sim 2^{n} \frac{l_{g}+l_{b}}{l_{t}}\right)>0 \text {. }
$$

By extracting the maximal value of $n$ to which (3.6) applies, we obtain $N_{s}$, the number of sheets per coalesced region as

$$
\begin{aligned}
N_{s} & =2^{\text {floor }\left[1+\ln \left(2 \pi \sqrt{\left.4 L(0) \cos (\theta) \varepsilon_{2}^{2} / C_{a}-L(0)^{2} G / 2\right)} / \ln 4\right]\right.} \\
& =2^{\text {floor }\left[1+\ln \left(2 \pi \sqrt{\left(4 \gamma \cos \theta / l_{g}-\rho g l(0) / 2\right) l(0) l_{z} l_{t}^{3} / 3 E I l_{g}}\right) / \ln 4\right]}
\end{aligned}
$$

where floor is rounding into the integer closer to zero. Hence, $N_{s}$ is limited to the subset $2^{N}$ where $N$ is the set of natural numbers and we thus obtain previously unreported relations between $N_{s}$ and gravity, $G$, as well as the initial length of the wetted region $L(0)$ (which is affected by the extraction process and the liquid viscosity).

\subsection{Experimental illustration of the theoretical results}

We preformed experiments to illustrate the theoretical results obtained from our analysis (3.7). Specifically we illustrate the multistep coalescence process, influence of gravity, and influence of the extraction process on the elasto-capillary instability. Our experimental setup consists of 100 plastic sheets which are extracted from a liquid bath by a linear stage actuator (Thorlabs ${ }^{\mathrm{TM}}$ LNR50SEK1) at a constant speed. We focus on cases in which the extraction speed is at least an order-of-magnitude faster or slower than the characteristic liquid speed, which enables estimating the value of $l(0)$ (see $\S 3.1$ ). After computing $l(0)$ the theoretical value of $N_{s}$ can be calculated via (3.7) and compared with the experimental value. The geometric and physical parameters of the plastic sheets (defined in figure 1) are thickness of $l_{b}=1.1 \times 10^{-4} \mathrm{~m}$, width in the $z$-direction $l_{z}=7.62 \times 10^{-2} \mathrm{~m}$, gap between the sheets of $l_{g}=10^{-3} \mathrm{~m}$, second moment of inertia of $I=8.45 \times 10^{-15} \mathrm{~m}^{4}$, and Young's modulus of $E=7.93 \times 10^{9} \mathrm{~Pa}$. The length of the sheets $l_{t}$ can be modified. The liquids used are deionized water (viscosity $\mu=10^{-3} \mathrm{~Pa} \mathrm{~s}$, density $\rho=10^{3} \mathrm{~kg} \mathrm{~m}^{-3}$, surface tension $\gamma=0.072 \mathrm{~N} \mathrm{~m}^{-1}$, and wetting angle $\theta \approx 70^{\circ}$ ) and glycerol (viscosity $\mu=1.41 \mathrm{~Pa} \mathrm{~s}$, density $\rho=1261 \mathrm{~kg} \mathrm{~m}^{-3}$, surface tension $\gamma=0.064 \mathrm{~N} \mathrm{~m}^{-1}$ and wetting angle $\left.\theta \approx 70^{\circ}\right) . N_{s}$ is the mean number 


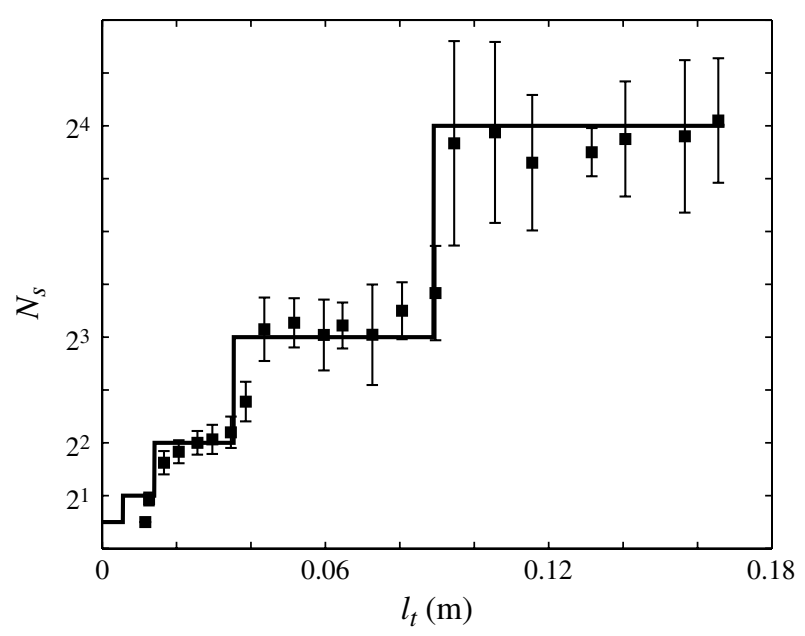

FIGURE 3. Length of the sheets $l_{t}$ versus the number of collapsed sheets per coalesced region $N_{s}$. The solid line is the theoretical solution (3.7), the square symbols represent the experimental data and error bars mark one standard deviation. The geometric and physical parameters are $l_{b}=1.1 \times 10^{-4} \mathrm{~m}, l_{z}=7.62 \times 10^{-2} \mathrm{~m}, l_{g}=10^{-3} \mathrm{~m}, I=8.45 \times 10^{-15} \mathrm{~m}^{4}$, and $E=7.93 \times 10^{9} \mathrm{~Pa}$. The liquid used is deionized water.

of sheets per coalesced region and is measured by dividing the total number of sheets $N$ by the number of bundles created by elasto-capillary coalescence.

Figure 3 compares $l_{t}$, the length of the elastic sheets, versus $N_{s}$, the mean number of sheets per coalesced region. The solid line is the theoretical solution from (3.7), the square symbols mark the experimental data and the error bars mark one standard deviation. The liquid used is deionized water. The extraction speed $\partial l_{e} / \partial t \approx 10^{-3} \mathrm{~m} \mathrm{~s}^{-1}$ is small in comparison with the characteristic speed of the flow due to capillary forces, readily estimated as $\gamma l_{g} / 12 \mu l(0)\left(=1.5 \mathrm{~m} \mathrm{~s}^{-1}\right)$, so that $l(0)$ is determined by quasi-static capillary rise as $l(0)=2 \gamma \cos (\theta) / l_{g} \rho g\left(\approx 5 \times 10^{-3} \mathrm{~m}\right)$ for all examined cases. With the exception of the transition regions (the locations where $N_{s}$ increases from $2^{n}$ to $2^{n+1}$ ), the mean number of sheets per coalesced region $N_{s}$ is within in the subset $2^{N}$, where $N$ of the set of natural numbers, as expected from our analysis. Furthermore, the experimental location of the transition regions agrees well with the theoretical calculations. These results are similar to the data obtained by Pokroy et al. (2009) (see figure $3 b$ in Pokroy et al. 2009).

Figure 4 illustrates the effect of gravity on elasto-capillary coalescence by presenting two identical configurations with gravity acting in opposing directions. The length of the sheets is $l_{t}=1.26 \times 10^{-2} \mathrm{~m}$ and the liquid used is glycerol. The extraction speed $\partial l_{e} / \partial t \approx 5 \times 10^{-3} \mathrm{~m} \mathrm{~s}^{-1}$ is large in comparison with the characteristic speed of the flow due to capillary forces, readily estimated as $\gamma l_{g} / 12 \mu l(0)\left(=10^{-3} \mathrm{~m} \mathrm{~s}^{-1}\right)$. Therefore the influence of flow during the extraction process on the value of $l(0)$ can be neglected and $l(0)$ is determined from its value before the extraction, defined as $l(0)=2 \gamma \cos (\theta) / l_{g} \rho g+l_{t}-l_{e}(0) \approx 2.6 \times 10^{-3} \mathrm{~m}$ (see figure 1). Equation (3.7) predicts that gravity (acting in the direction defined in figure 1) inhibits the elastocapillary instability and can thus affect the value of $N_{s}$. It is not possible to perform the experiment upside down; however, for the case of small values of $l_{t}$ corresponding to $N_{s}=2^{0}$, no coalescence occurs (see figure $4 a$ ). This enables us 


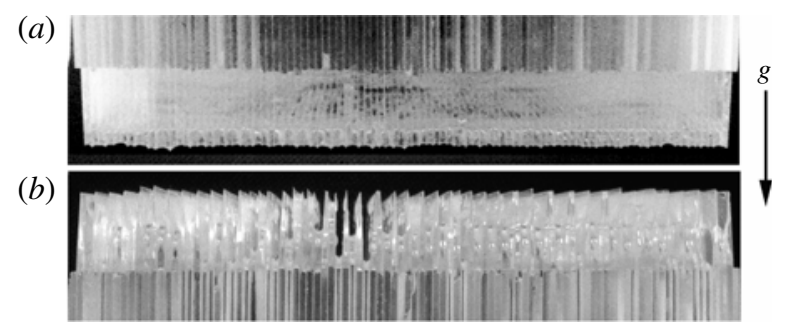

FIGURE 4. The influence of gravity direction on $N_{s}$, the mean number of sheets per coalesced region: $(a)$ gravity pointing toward the unclamped end of the sheets; $(b)$ gravity pointing toward the clamped end of the sheets. The liquid used is glycerol. The geometric and physical parameters are $l_{b}=1.1 \times 10^{-4} \mathrm{~m}, l_{z}=7.62 \times 10^{-2} \mathrm{~m}, l_{g}=10^{-3} \mathrm{~m}, I=8.45 \times 10^{-15} \mathrm{~m}^{4}$, $E=7.93 \times 10^{9} \mathrm{~Pa}$ and $l_{t}=1.26 \times 10^{-2} \mathrm{~m}$.

to rotate the configuration and compare two identical cases with gravity acting in opposing directions. In figure $4(a)$ we observe $N_{s}=2^{0}$ for the case of gravity pointing toward the unclamped end of the sheets, and for gravity pointing in the opposing direction (figure $4 b$ ) $N_{s}$ has a mean value of 1.98 and standard deviation of 0.56 , corresponding to $N_{s}=2^{1}$. From (3.7) we expect that, for the given parameters, gravity would change $N_{s}$ from $2^{0}$ to $2^{1}$ within the range of $l_{t}=6.3 \times 10^{-3}$ to $7.2 \times 10^{-3} \mathrm{~m}$. Experimentally this region is found to be around $l_{t} \approx 1 \times 10^{-2} \mathrm{~m}$, significantly different from the theoretically estimated values. This error is due to the $O\left(\varepsilon_{1}\right)$ accuracy of our calculations and the relatively large value of $\varepsilon_{1} \approx 0.25$ for the small value of $l_{t}$ examined.

Figure 5 illustrates the effect of extraction speed by presenting the coalescence of two identical configurations which have been extracted from the liquid at different constant speeds. The liquid used is glycerol, the length of the sheets is $l_{t}=3.46 \times 10^{-2} \mathrm{~m}$ and gap between the liquid and the clamped end of the sheets is $l_{e}(0)=1.96 \times 10^{-2} \mathrm{~m}$ (see figure 1 ). The characteristic speed of the flow due to capillary forces is $\gamma l_{g} / 12 \mu l(0) \approx 10^{-3} \mathrm{~m} \mathrm{~s}^{-1}$. Figure $5(a)$ presents the coalescence for an extraction speed of $10^{-5} \mathrm{~m} \mathrm{~s}^{-1}$, small in comparison with the characteristic speed due to capillary and viscous forces. In this case $l(0)$ is determined by quasistatic capillary rise and can be estimated as the initial length of the liquid-filled region $l(0)=2 \gamma \cos (\theta) / l_{g} \rho g \approx 5 \times 10^{-3} \mathrm{~m}$. Figure $5(b)$ presents the coalescence for an extraction speed of $5 \times 10^{-3} \mathrm{~m} \mathrm{~s}^{-1}$, large in comparison with the characteristic speed due to capillary forces. In this case the flow during the extraction can be neglected and $l(0)$ can be estimated as $l(0)=2 \gamma \cos (\theta) / l_{g} \rho g+l_{t}-l_{e}(0) \approx 1.5 \times 10^{-2} \mathrm{~m}$ (see figure 1). Changing the value of $l(0)$ affects the value of $N_{s}$ obtained from (3.7), which is $N_{s}=2^{2}$ and $2^{3}$ for $l(0)=5 \times 10^{-3}$ and $1.5 \times 10^{-2} \mathrm{~m}$, respectively. From figure $5(a)$ we observe that for an extraction speed of $10^{-5} \mathrm{~m} \mathrm{~s}^{-1} N_{s}$ has the mean value of 4.8 and standard deviation of 1.05 , corresponding to $N_{s}=2^{2}$ and for extraction speed of $5 \times 10^{-3} \mathrm{~m} \mathrm{~s}^{-1}$ the mean value of $N_{s}$ is 7.4 and standard deviation of 1.85 , corresponding to $N_{s}=2^{3}$. Hence, the experimental data are in agreement with the theoretical solution (3.7).

\section{Concluding remarks}

In the existing literature elasto-capillary coalescence is studied via minimal energy analysis of the capillary and elastic energies of the post-coalescence state, yielding 
(a)

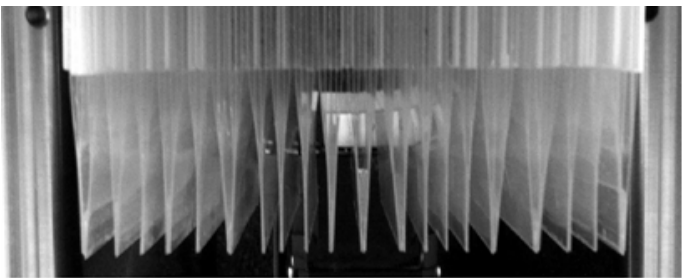

(b)

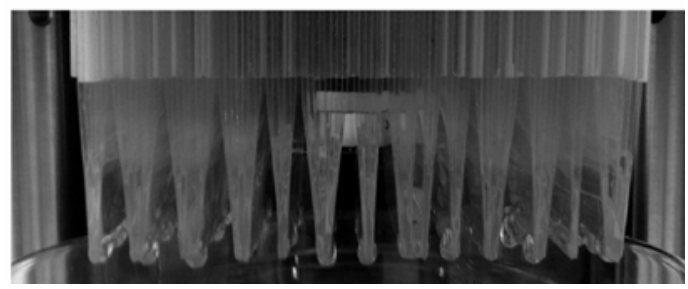

FIgURE 5. The influence of extraction speed $\partial l_{e} / \partial t$ on $N_{s}$, the number of sheets per coalesced region: (a) $10^{-5} \mathrm{~m} \mathrm{~s}^{-1}$; (b) $5 \times 10^{-3} \mathrm{~m} \mathrm{~s}^{-1}$. The liquid is glycerol. The geometric and physical parameters are $l_{b}=1.1 \times 10^{-4} \mathrm{~m}, l_{z}=7.62 \times 10^{-2} \mathrm{~m}, l_{g}=10^{-3} \mathrm{~m}, I=$ $8.45 \times 10^{-15} \mathrm{~m}^{4}, E=7.93 \times 10^{9} \mathrm{~Pa}$ and $l_{t}=3.46 \times 10^{-2} \mathrm{~m}$.

the maximal stable post-coalescence bundle size. An assumption then is made that coalescence will continue as long as it will not create a bundle size larger than the maximal stable post-coalescence bundle size.

We studied the stability of the system before the coalescence occurred and obtained previously unreported relations between the extraction process, viscous forces, body forces and the coalescence instability, thus undermining the assumption that coalescence will occur as long as it will not create a bundle larger than the maximal stable post-coalesced size. The fastest growing instability was found to correspond with the minimal possible wavelength which is two sheets before any coalescence occurred, or $2^{n+1}$ sheets after $n$ coalescences. This, in turn, was found to determine the multistep hierarchical structure of elasto-capillary coalescence and to limit the mean number of sheets per coalesced region, $N_{s}$, to the subset $2^{N}$ where $N$ is the set of natural numbers.

We performed experiments to illustrate our theoretical results. The limitation of $N_{s}$ to the subset $2^{N}$ as well as the relation between $N_{s}$ and $l_{t}$ were experimentally examined in figure 3 , the influence of gravity was examined in figure 4 , and the influence of the extraction speed (and thus $l(0)$ ) was examined in figure 5 . The limitation of $N_{s}$ to the subset $2^{N}$ is also supported by the experimental data of Pokroy et al. (2009) (see figure 3 in Pokroy et al. 2009) and Chandra \& Yang (2010) (see figure 10 in Chandra \& Yang 2010) examining elasto-capillary coalescence of pillars. The work of Pokroy et al. (2009) clearly presents the transitions for $N_{s}=2^{n}$ to $2^{n+1}$. In the work of Chandra \& Yang (2010) all of the measured values of $N_{s}$ fit into the subset $2^{N}$ (within accuracy of one standard deviation). However, the transitions from $N_{s}=2^{n}$ to $2^{n+1}$ are not evident due to the small sample size of three in figure $10 \mathrm{c}$ and 4 in figure $10 d$ (both in Chandra \& Yang 2010).

\section{Acknowledgements}

The authors wish to acknowledge the work of Aria Vahdani on the experimental setup and also wish to acknowledge the support of Charyk Laboratory. 


\section{REFERENCES}

Antkowiak, A., Audoly, B., Josserand, C., Neukirch, S. \& Rivetti, M. 2011 Instant fabrication and selection of folded structures using drop impact. Proc. Natl Acad. Sci. 108, 10400-10404.

Aristoff, J. M., Duprat, C. \& Stone, H. A. 2011 Elastocapillary imbibition. Intl J. Non-Linear Mech. 46, 648-656.

Bico, J., Roman, B., Moulin, L. \& Boudaoud, A. 2004 Elastocapillary coalescence in wet hair. Nature 432, 690.

Boudaoud, A., Bico, J. \& Roman, B. 2007 Elastocapillary coalescence: aggregation and fragmentation with a maximal size. Phys. Rev. E 76, 060102.

CHANDRA, D. \& YANG, S. 2010 Stability of high-aspect-ratio micropillar arrays against adhesive and capillary forces. Acc. Chem. Res. 43, 1080-1091.

Chandra, D., Yang, S., Soshinsky, A. A. \& Gambogi, R. J. 2009 Biomimetic ultrathin whitening by capillary-force-induced random clustering of hydrogel micropillar arrays. ACS Appl. Mater. Interfaces 1, 1698-1704.

Chiodi, F., Roman, B. \& Bico, J. 2010 Piercing an interface with a brush: Collaborative stiffening. Europhys. Lett. 90, 044006.

De Gennes, P. G., Brochard-Wyart, F., Quéré, D., Reisinger, A. \& Widom, B. 2004 Capillarity and Wetting Phenomena: Drops, Bubbles, Pearls, Waves. Springer.

De Volder, M., Tawfick, S. H., Park, S. J., Copic, D., Zhao, Z., Lu, W. \& Hart, A. J. 2010 Diverse 3D microarchitectures made by capillary forming of carbon nanotubes. Adv. Mater. 22, 4384-4389.

De Volder, M. F. L., Park, S. J., Tawfick, S. H., Vidaud, D. O. \& Hart, A. J. 2011 Fabrication and electrical integration of robust carbon nanotube micropillars by self-directed elastocapillary densification. J. Micromech. Microengng 21, 045033.

DuAn, H. \& BergGren, K. K. 2010 Directed self-assembly at the $10 \mathrm{~nm}$ scale by using capillary force-induced nanocohesion. Nano Lett. 10, 3710-3716.

Duprat, C., Aristoff, J. M. \& Stone, H. A. 2011 Dynamics of elastocapillary rise. J. Fluid Mech. 679, 641-654.

Duprat, C., Protire, S., Beebe, A. Y. \& Stone, H. A. 2012 Wetting of flexible fibre arrays. Nature 482, 510-513.

Elwenspoek, M., Abelmann, L., Berenschot, E., van Honschoten, J., Jansen, H. \& TAS, N. 2010 Self-assembly of (sub-)micron particles into supermaterials. J. Micromech. Microengng 20, 064001.

Gat, A., NAvaz, H. \& GHARIB, M. 2011 Dynamics of freely moving plates connected by a shallow liquid bridge. Phys. Fluids 23 (9), 097101+.

Huang, X., Zhou, J., Sansom, E., Gharib, M. \& Haur, S. C. 2007 Inherent opening controlled pattern formation in carbon nanotube arrays. Nanotechnology 18, 305301.

Journet, C., Moulinet, S., Ybert, C., Purcell, S. T. \& Bocquet, L. 2005 Contact angle measurements on superhydrophobic carbon nanotube forests: effect of fluid pressure. Europhys. Lett. 71 (1), 104-109.

Kang, S. H., Wu, N., Grinthal, A. \& Aizenberg, J. 2011 Meniscus lithography: evapouration-induced self-organization of pillar arrays into moir patterns. Phys. Rev. Lett. 107, 177802.

Kim, H.-Y. \& Mahadevan, L. 2006 Capillary rise between elastic sheets. J. Fluid Mech. 548, $141-150$.

LeAL, G. 2007 Advanced Transport Phenomena: Fluid Mechanics and Convective Transport Processes. Cambridge Series in Chemical Engineering. Cambridge University Press.

LiU, J.-L., Feng, X.-Q., XIA, R. \& ZhaO, H.-P. 2007 Hierarchical capillary adhesion of microcantilevers or hairs. J. Phys. D: Appl. Phys. 40, 5564-5570.

Pineirua, M., Bico, J. \& Roman, B. 2010 Capillary origami controlled by an electric field. Soft Matt. 6, 4491-4496.

Pokroy, B, Kang, S. H., Mahadevan, L. \& Aizenberg, J. 2009 Self-organization of a mesoscale bristle into ordered, hierarchical helical assemblies. Science 323, 237-240. 
Py, C., Bastien, R., Bico, J., Roman, B. \& Boudaoud, A. $2007 a$ 3D aggregation of wet fibres. Europhys. Lett. 77, 44005.

Py, C., Reverdy, P., Doppler, L., Bico, J., Roman, B. \& Baroud, C. N. 2007b Capillary origami: spontaneous wrapping of a droplet with an elastic sheet. Phys. Rev. Lett. 98, 156103.

Sikalo, S., Tropea, C. \& Ganic, E. 2005 Dynamic wetting angle of a spreading droplet. Exp. Therm. Fluid Sci. 29, 795.

TARoni, M. \& Vella, D. 2012 Multiple equilibria in a simple elastocapillary system. J. Fluid Mech. 712, 273-294.

ZhaO, Y.-P. \& FAn, J.-G. 2006 Clusters of bundled nanorods in nanocarpet effect. Appl. Phys. Lett. 88, 103123.

Zhao, Z., Tawfick, S. H., Park, S. J., De Volder, M., Hart, A. J. \& LU, W. 2010 Bending of nanoscale filament assemblies by elastocapillary densification. Phys. Rev. E 82, 041605. 